\title{
Azlocillin Sodium
}

National Cancer Institute

\section{Source}

National Cancer Institute. Azlocillin Sodium. NCI Thesaurus. Code C65243.

The sodium salt form of azlocillin, a semisynthetic, extended spectrum acylampicillin with antibacterial activity. Azlocillin binds to penicillin-binding proteins (PBPs) located inside the bacterial cell wall, thereby inhibiting the cross-linkage of peptidoglycans, which are critical components of the bacterial cell wall. This prevents proper bacterial cell wall synthesis, thereby results in the weakening of the bacterial cell wall and eventually leading to cell lysis. 